Current issues in adjuvant treatment of stage II colon cancer

Peer-reviewed author version

André, Thierry; Sargent, D; Tabernero, J; O'Connell, M; BUYSE, Marc; Sobrero, A;

Misset, J.L.; Boni, C \& de Gramont, A (2006) Current issues in adjuvant treatment of stage II colon cancer. In: ANNALS OF SURGICAL ONCOLOGY, 13(6). p. 887-898.

DOI: $10.1245 /$ ASO.2006.07.003

Handle: http://hdl.handle.net/1942/1572 


\title{
Current Issues in Adjuvant Treatment of Stage II Colon Cancer
}

\author{
Thierry André, MD,${ }^{1,2,3}$ Daniel Sargent, $\mathrm{PhD},{ }^{4}$ Josep Tabernero, $\mathrm{MD},{ }^{5}$ \\ Michael O'Connell, MD, ${ }^{6}$ Marc Buyse, $\mathrm{ScD},{ }^{7}$ Alberto Sobrero, MD, ${ }^{8}$ \\ Jean-Louis Misset, MD, ${ }^{9}$ Corrado Boni, MD,${ }^{10}$ and Aimery de Gramont, $\mathrm{MD}^{2,3,11}$
}

\author{
${ }^{1}$ Service d'Oncologie Médicale, Hôpital Tenon, 4 Rue de la Chine, 75970 Paris Cedex 20, France \\ ${ }^{2}$ CancerEst, Hôpital Saint Antoine, 184 Rue du Faubourg Saint Antoine, 75571 Paris Cedex 12, France \\ ${ }^{3}$ GERCOR (Franch Oncology Research Group), 22 Rue Malher, 75004 Paris, France \\ ${ }^{4}$ Mayo Clinic, 200 First Street SW, Rochester, Minnesota 55905 \\ ${ }^{5}$ Vall d'Hebron University Hospital, P Vall d'Hebron 119-129, 08035 Barcelona, Spain \\ ${ }^{6}$ NSABP Foundation Research Program, 4 Allegheny Center, Pittsburgh, Pennsylvania 15212 \\ ${ }^{7}$ IDDI, 430 Avenue Louise, BP 14, B 1050 Brussels, Belgium \\ ${ }^{8}$ Ospedale S. Martino, Largo Benzi 10, Genova 16132, Italy \\ ${ }^{9}$ Hôpital Saint-Louis, 1 Avenue Claude Vellefaux, 75010 Paris, France \\ ${ }^{10}$ Arcispedale S. Maria Buova, Viale Resorgimento 80, Regio Emilia 42100, Italy \\ ${ }^{11}$ Hôpital Saint-Antoine, 184 Rue du Faubourg Saint Antoine, 75571 Paris Cedex 12, France
}

\begin{abstract}
Background: Adjuvant chemotherapy with 5-fluorouracil modulated by folinic acid, combined with oxaliplatin, has now become an accepted standard of care for patients with stage III colon cancer. In contrast, the use of adjuvant therapy for stage II patients remains controversial, and the identification of reliable prognostic factors to aid therapeutic decision making is crucial.

Methods: The authors critically review the results of clinical trials and meta-analyses investigating the value of adjuvant chemotherapy for stage II patients, emphasizing the heterogeneous nature of this population and the difficulty of performing clinical trials with sufficient power to reliably assess treatment efficacy. They also discuss the evidence concerning potential prognostic factors, particularly molecular markers.

Results: Available clinical trial data do not support the routine use of adjuvant chemotherapy for all stage II patients but suggest that it should be considered, particularly for certain high-risk patients. Recent guidelines advocate considering factors such as tumor differentiation, tumor perforation, number of lymph nodes examined, and $\mathrm{T}$ stage when assessing the likely benefit:risk ratio. Microsatellite instability and allelic imbalance seem to be strong predictors of good and poor prognosis, respectively, and in the near future, therapeutic decisionmaking models are likely to be further refined by the inclusion of such molecular markers.

Conclusions: There is growing evidence that the prognosis of certain stage II patients with unfavorable prognostic factors can be improved by adjuvant chemotherapy, and increasingly refined tools are now available to define those most likely to benefit. Referral of stage II patients for individual assessment is strongly recommended.
\end{abstract}

Received July 5, 2005; accepted November 15, 2005; published online April 14, 2006.

Address correspondence and reprint requests to: Thierry André, MD, E-mail: thierry.andre@tnn.aphp.fr.

Published by Springer Science+Business Media, Inc. @ 2006 The Society of Surgical Oncology, Inc. 
Key Words: Colon cancer, stage II-Adjuvant chemotherapy-5-Fluorouracil—LeucovorinOxaliplatin.

Over the past years, considerable progress has been made in the treatment of patients with colon cancer. The use of optimized folinic acid (FA)-modulated 5fluorouracil (5-FU) regimens has increased the median survival of patients with metastatic (stage IV) colon cancer from barely 6 months without treatment to $>12$ months. ${ }^{1}$ The addition of new chemotherapeutic agents (e.g., oxaliplatin and irinotecan) to these regimens has further extended the median survival to approximately 20 months. ${ }^{2-7}$ The combination of newly approved biological agents, such as bevacizumab and cetuximab, with optimal chemotherapy promises to prolong survival still more. ${ }^{8,9}$

At diagnosis, approximately $14 \%$ of patients have stage I disease, $28 \%$ have stage II, $37 \%$ have stage III, and $21 \%$ have stage IV. ${ }^{10}$ In the context of continually improving treatments for advanced disease, it is not surprising that the investigation of newer chemotherapies has been extended to patients with stage II and III colon tumors after surgical resection. In these patients, the prognosis varies widely according to the disease stage and, in particular, the extent of lymph node involvement and the extent to which the tumor has invaded the bowel wall (expressed by the $\mathrm{T}$ [tumor] and $\mathrm{N}$ [node] stage in the tumor-node-metastasis classification). The 5-year survival rate decreases from $>90 \%$ for stage I tumors to $50 \%$ for stage III tumors. ${ }^{11}$ Patients with stage II colon cancer constitute a particularly heterogeneous population. Patients with tumors that are barely starting to penetrate through the bowel wall have a prognosis approaching that of patients with stage I cancer. In contrast, similarly staged patients with aggressive and extensive tumors showing extramural venous spread and involvement of the serosa or adjacent organs have a prognosis similar to that of patients with stage III cancer. $^{12}$

In view of the generally good prognosis of patients with resected stage I tumors, clinical studies on adjuvant chemotherapy have mainly focused exclusively on patients with resected stage III tumors or on patients with stage II or stage III tumors but without differentiating between these two groups. Trials performing separate analyses of the effect of adjuvant therapy on stage II and stage III patients tend to have insufficient power to assess treatment efficacy adequately in stage II patients, because the number of these patients is generally small and the event rate is low. It has been estimated that a prospective clinical trial of adjuvant chemotherapy including a nontreatment control arm and designed to detect a $4 \%$ survival benefit at 5 years among stage II colon cancer patients with a baseline 5-year survival prognosis of $75 \%$ would require at least 4700 patients. ${ }^{13}$ No study of this size has yet been published.

On the basis of published data on patients with stage III cancer, 6 months of adjuvant chemotherapy with 5-FU/FA became the standard of care for such patients in the early 1990s. ${ }^{14-18}$ More recently, the addition of oxaliplatin to 5-FU/FA has further improved patient outcomes, thus establishing this combination as a new standard of care. ${ }^{19,20}$ Although the benefit of adjuvant chemotherapy for stage III colon cancer is now acknowledged, the use of adjuvant therapy in patients with stage II colon cancer remains controversial. Increasing attention is being focused on the identification of unfavorable prognostic factors that could aid in making the decision for or against treatment on the basis of the relative benefits and risks for each individual patient. This review presents an overview of the recent and extensive published data concerning adjuvant chemotherapy for stage II colon cancer.

\section{EFFICACY OF ADJUVANT CHEMOTHERAPY IN STAGE II COLON CANCER: ANALYSES, META-ANALYSES, AND RECENT CLINICAL STUDIES}

Published clinical trials conducted until very recently were not been large enough to demonstrate a statistically significant advantage for chemotherapy in stage II colon cancer. For this reason, several analyses and meta-analyses have been performed of clinical trials comparing adjuvant therapy with observation in patients with stage II colon or colorectal cancer.

The National Surgical Adjuvant Breast and Bowel Project (NSABP) performed an analysis of the results of its four trials on adjuvant chemotherapy in patients with colon cancer: two comparing surgery alone with surgery plus adjuvant chemotherapy, and two comparing different adjuvant chemotherapy regimens. ${ }^{21} \mathrm{~A}$ total of 3820 patients were available for 
analysis: $41 \%$ had stage II and 59\% had stage III disease. Two pooled treatment groups were compared within each stage of disease: one comprising the less effective treatment group from each trial and the other comprising the more effective group. The relative reduction in risk in the more effective treatment group was $30 \%$ for the 1565 patients with stage II colon cancer (irrespective of the presence or absence of adverse prognostic factors), compared with $18 \%$ for the 2255 patients with stage III colon cancer. In two of the trials, the risk reduction achieved in stage II patients was statistically significant. However, this analysis has been criticized for pooling the results from trials with different control and treatment arms.

The International Multicentre Pooled Analysis of B2 Colon Cancer Trials (IMPACT B2) included data on 1016 patients with stage II colon cancer generated in 5 randomized clinical trials comparing 5-FUcontaining adjuvant chemotherapy with no treatment. $^{22}$ This analysis found 5 -year overall survival (OS) and disease-free survival (DFS) rates of $80 \%$ and $73 \%$, respectively, in untreated patients versus $82 \%$ and $76 \%$, respectively, in treated patients, corresponding to relative risk reductions of $17 \%$ for DFS and $14 \%$ for OS with adjuvant treatment. Although these differences failed to reach statistical significance, there was a trend toward a small benefit in absolute survival with adjuvant therapy (2\%; one sided). In the multivariable Cox analysis, age and tumor grade were the only independent predictors for OS and DFS. In the population analyzed, $1.5 \%$ of the patients had T4 tumors (defined as tumors directly invading an adjacent organ or perforating the visceral peritoneum), and $11 \%$ had poorly differentiated tumors. The prognostic factors analyzed did not include perforation, bowel obstruction, venous invasion, or the number of lymph nodes examined. One of the major criticisms of this meta-analysis is the relatively small number of cases included. Another meta-analysis of 3499 patients participating in 10 studies evaluating the efficacy of short continuous portal vein infusion of chemotherapy demonstrated that the relative risk benefit of this treatment on the risk of death was nearly identical in stage III and in stage I/II patients. ${ }^{23}$

More recently, a pooled analysis of seven randomized trials comparing adjuvant 5-FU/FA or 5-FU/levamisole with surgery alone in stage II and III colon cancer (including the five IMPACT B2 trials) was conducted, with the aim of clarifying the benefit of adjuvant therapy in specific subgroups of patients. ${ }^{24}$ This pooled analysis showed a significant improvement in 5-year DFS in the 1440 patients who presented with node-negative disease (76\% vs. $72 \%$ for node-positive disease; $P=.0490)$. However, the difference in 5-year OS $(81 \%$ vs. $80 \%$, respectively) did not reach statistical significance. A significant interaction between stage and treatment was evident, and adjuvant chemotherapy benefited stage III patients to a greater extent than stage II patients. Significant prognostic factors in this analysis, besides nodal status, included histological tumor grade and depth of tumor invasion into the bowel wall. Other prognostic factors (e.g., perforation, bowel obstruction, venous invasion, and number of lymph nodes examined) were not evaluated in this study.

In an attempt to help physicians assess the benefit:risk ratio of adjuvant chemotherapies for each individual patient, tools have been developed that incorporate the $\mathrm{T}$ and $\mathrm{N}$ stage with age, tumor differentiation, and tumor perforation, in addition to the number of lymph nodes retrieved. ${ }^{24} \mathrm{~A}$ model incorporating these factors is now available to provide physicians with tailored estimates of 5-year DFS and OS probabilities with surgery alone and with surgery plus 5-FU-based adjuvant chemotherapy. ${ }^{25}$

The Cancer Care Ontario Program in EvidenceBased Care conducted two systematic reviews of data from clinical trials comparing adjuvant therapy with observation in patients with resected stage II colon or colorectal cancer. ${ }^{26,27}$ The more recent of these reviewed 37 randomized controlled trials and 11 metaanalyses that included a total of 20,317 patients (7,803 with colon cancer and 12,514 with colorectal cancer). The proportion of patients with stage II disease varied from $23 \%$ to $100 \% .^{27}$ A meta-analysis was conducted on the data obtained on stage II patients whenever available (4187 patients in 18 trials). Data were selected on the basis of stringent criteria that required inclusion of a surgery-alone control arm and 5-FU-based adjuvant chemotherapy (16 trials) or immunotherapy alone ( 2 trials). The relative risk reduction in mortality across the trials was .87 (hazard ratio [HR], .87; 95\% confidence interval [CI], $.75-1.01 ; P=.07)$; however, this result should be interpreted cautiously because the trials used a variety of adjuvant therapies.

Several studies have investigated adjuvant therapy with oral fluoropyrimidines. The first clinical trials comparing surgery followed by adjuvant therapy with oral fluoropyrimidines versus surgery alone were conducted in Japan, where the development of adjuvant chemotherapy for colorectal cancer was essentially based on these agents. A meta-analysis of 
three of these trials - all conducted by the Japanese Foundation for the Multidisciplinary Treatment for Cancer and using separate randomizations for patients with colon and rectal cancer-was recently published. ${ }^{28}$ The total population analyzed comprised 5233 patients: 2848 (54\%) presented with colon cancer and 2385 (46\%) with rectal cancer. Overall, 2295 patients (44\%) had stage II disease, and 2348 (45\%) had stage III disease. Both OS (HR, .89; $P=$ $.04)$ and DFS (HR, .85; $P<.001)$ were statistically significantly increased by adjuvant chemotherapy in the population as a whole, with a similar benefit observed in patients with colon and rectal tumors. Five-year survival was increased by $3.7 \%, 4.3 \%$, and $2.4 \%$ in patients with stage I, II, and III tumors, respectively.

The Netherlands Adjuvant Colorectal Cancer Project trial reported an improved 5-year survival rate in stage II colon and rectal cancer patients receiving adjuvant 5-FU plus levamisole (78\% vs. $70 \%$ ) but did not specify the level of statistical significance for the difference observed (no subgroup analyses). ${ }^{29}$

The recently completed Quick And Simple And Reliable (QUASAR) trial, not included in either published meta-analyses or the Cancer Care Ontario Program in Evidence-Based Care review and so far published only in abstract form, compared adjuvant chemotherapy (5-FU modulated by FA with or without levamisole) with observation in patients with resected colon or rectal cancer. ${ }^{30}$ A total of 3238 patients were enrolled, of whom $91 \%$ presented with stage II disease (71\% colon and $29 \%$ rectal cancer). Updated results presented at the American Society of Clinical Oncology (ASCO) 2004 meeting (median follow-up, 4.6 years) showed a significantly improved 5 -year OS $(80.3 \%$ vs. $77.4 \%$, respectively; $P=.02)$ and a significantly lower 5-year recurrence rate (22.2\% vs. $26.2 \%$, respectively; $P=.001)$ in the adjuvant chemotherapy group compared with observation. Separate analysis of stage II patients showed a significant reduction in the number of deaths with adjuvant chemotherapy (224 vs. 262, respectively; $P=.04$ ). This is the largest single study in the population of stage II patients, and, if these preliminary results are confirmed in the subsequent publication, it will be the first to demonstrate a statistically significant advantage of adjuvant chemotherapy for this group. The analysis of prognostic variables in this study should help to identify which subgroups of stage II patients are likely to benefit the most from adjuvant chemotherapy.
An analysis conducted between 1991 and 1996 in 3151 US Medicare patients aged between 65 and 75 years with resected stage II colon cancer revealed that $27 \%$ received adjuvant chemotherapy, compared with $75 \%$ of stage III patients. ${ }^{31}$ In that study, a younger age at diagnosis, white race, low comorbidity, and poorly differentiated tumor histological characteristics were all associated with a greater likelihood of being treated, whereas sex, socioeconomic status, and the number of lymph nodes examined had no bearing on the treatment rate. The 5-year survival rate was $78 \%$ for the treated patients and $75 \%$ for those not treated. After adjusting for known differences between the groups, the HR for survival associated with adjuvant treatment was .91 (95\% CI, .77-1.09), which is consistent with the clinical trial data discussed previously.

When viewed as a whole, the results of all these studies (clinical studies, analyses, and meta-analyses) demonstrate remarkable consistency. The repeated finding is that 5-FU-based adjuvant therapy provides a small but real benefit in terms of DFS and OS rates. The physician and patient, when fully armed with this knowledge, must then make a decision regarding the relative importance of the very modest benefit in prognosis when weighed against the risks, toxicity, and inconvenience of therapy.

\section{EFFECT OF NEW CHEMOTHERAPEUTIC AGENTS ON STAGE III ONLY OR BOTH STAGE II AND STAGE III COLON CANCER}

Two studies that were not designed to test the value of chemotherapy for patients with stage II colon cancer compared oral fluoropyrimidines with a 5-FU/FA bolus regimen. These two studies were presented at the ASCO meeting in 2004. The first compared capecitabine with the Mayo clinic regimen (monthly bolus regimen of 5-FU/FA) in patients with stage III cancer only. The HR for DFS at 3 years was .87 (95\% CI, .751). The primary end point of this study was met, thus demonstrating that capecitabine is not inferior to the Mayo Clinic regimen (in fact, there was even a trend to superiority for capecitabine). ${ }^{32}$ The second study, conducted by the NSABP (C06 study) in patients with stage II and III colon cancer, compared oral uracil and tegafur + FA to the Roswell Park regimen (weekly bolus regimen of 5-FU/FA) and demonstrated the perfect equality of uracil and tegafur + FA and the Roswell Park regimen (in terms of DFS and OS at 5 years). ${ }^{33}$ No analysis concerning only patients with stage II colon cancer was performed. ${ }^{33}$ 
The Multi-Center International Study of Oxaliplatin/5-Fluorouracil/Leucovorin in the Adjuvant Treatment of Colon Cancer study compared the benefit of standard 5-FU/FA adjuvant chemotherapy (LV5FU2) with that of the same regimen but with the addition of oxaliplatin (FOLFOX4) in 2246 patients who had undergone curative resection for stage II or III colon cancer. ${ }^{19}$ After a median follow-up of 37.9 months, the 3-year DFS in the total population of stage II and III patients was $72.9 \%$ in the LV5FU2 arm and $78.2 \%$ in the FOLFOX4 arm (HR, .77; 95\% CI, .65-.91; $P=.002$ ), corresponding to a $23 \%$ reduction in the risk of relapse. Among the 899 patients with stage II disease, the addition of oxaliplatin resulted in a $20 \%$ decrease in risk of relapse, with an increase in the DFS rate from $84.3 \%$ to $87 \%$ after 3 years. ${ }^{34}$ An analysis of stage II patients with at least one adverse prognostic factor (T4 tumor, bowel obstruction or tumor perforation, poor differentiation, venous invasion, or $<10$ lymph nodes examined) was reported recently. In this subpopulation $(\mathrm{n}=576)$, FOLFOX4 patients had a $28 \%$ decrease in the risk of relapse (HR, .72; 95\% CI, .48-1.08) and a 3 -year DFS of $84.9 \%$ vs. $79.8 \%$ for LVSFU2 patients. These benefits were within the range of those observed in stage III patients and were associated with limited toxicity. No analysis of molecular markers was performed, because no samples were collected during the trial. ${ }^{34}$

In the NSABP C07 study, 2407 patients with stage II and III carcinoma of the colon (28.6\% stage II and $71.4 \%$ stage III) were randomized to receive either FULV (a 5-FU $500 \mathrm{mg} / \mathrm{m}^{2}$ bolus weekly and FA [leucovorin; LV] $500 \mathrm{mg} / \mathrm{m}^{2}$ weekly, each for 6 weeks of every 8-week cycle, for 3 cycles) or FLOX (same FULV regimen with the addition of oxaliplatin 85 $\mathrm{mg} / \mathrm{m}^{2}$ on weeks 1,3 , and 5 of each 8 -week cycle, for 3 cycles). The addition of oxaliplatin to the FULV regimen significantly improved the 3-year DFS in patients with stage II and III colon cancer (71.6\% vs. $76.5 \%$, respectively; $P<.004 ; \mathrm{HR}=.79 ; 95 \% \mathrm{CI}$, $.67-.93)$. No subgroup analysis was performed. The global test for interaction between treatment and tumor stage (II and III) was not significant $(P=.70) .{ }^{20}$

\section{PROGNOSTIC FACTORS IN STAGE II COLON CANCER}

It is increasingly recognized that not all stage II patients are likely to benefit equally from adjuvant chemotherapy, and the decision to treat patients is often made on the basis of perceived additional risk factors that may outweigh the inconvenience and likely toxicity of treatment. Despite the past uncertainty concerning the benefit:risk ratio of adjuvant chemotherapy in patients with stage II colon cancer, many of these patients nevertheless receive such treatment. Conversely, some centers deny chemotherapy to stage II patients because of the perceived lack of convincing data.

\section{Stage II Colon Cancer: A Heterogeneous Population}

Stage II colon cancer, characterized by the absence of lymph node involvement, covers a wide spectrum of disease, ranging from tumors with little penetration through the bowel wall to aggressive and extensive tumors with extramural venous spread and involvement of the serosa or adjacent organs. It is becoming increasingly evident that colon cancers are also heterogeneous with respect to many other clinical, pathologic, and biological factors and that traditional pathologic staging systems may be insufficient to predict outcome accurately. The proven value of adjuvant chemotherapy for patients with stage III colorectal cancer and the controversy surrounding its use in stage II patients have been a particular spur to the investigation of potential prognostic factors that enable identification of the patients most likely to benefit from this treatment.

With regard to stage II cancers, a wide variety of potential clinical and pathologic risk factors for recurrence have been investigated. The most important factors for predicting the risk of recurrence are emergency presentation (bowel perforation or occlusion), poorly differentiated tumor (histological grade), depth of tumor invasion and adjacent organ involvement (T4), extramural venous invasion, and peritoneal involvement. ${ }^{11,24,33}$ In the case of involvement of the resection margins, the resection is not curative. Age affects survival because of deaths from other causes; however, a benefit of adjuvant chemotherapy for both young and old patients was demonstrated in a large pooled analysis. ${ }^{35}$ In a study that specifically excluded patients with lymph node involvement (i.e., stage III patients), Petersen et al. ${ }^{36}$ identified six factors by univariate analysis that significantly influence prognosis $(P<.01)$. These were extent of tumor spread, peritoneal involvement, margin involvement, venous invasion, tumor perforation, and adjacent organ involvement. Patient age, sex, tumor site, and differentiation were not found to be statistically significant prognostic factors. The final validated Cox regression model combined four independent prognostic factors: peritoneal involve- 
TABLE 1. Colon cancer stages as defined by the American Joint Committee on Cancer (AJCC) staging manual (5th and 6th editions)

\begin{tabular}{llll}
\hline Stage & T stage & N stage & M stage \\
\hline AJCC 5th edition & & & \\
I & T1 or T2 & N0 & M0 \\
II & T3 or T4 & N0 & M0 \\
III & Any T & N1 & M0 \\
IV & Any T & Any N & M1 \\
AJCC 6th edition & & & \\
I & T1 or T2 & N0 & M0 \\
IIa & T3 & N0 & M0 \\
IIb & T4 & N0 & M0 \\
IIIa & T1 or T2 & N1 & M0 \\
IIIb & T3 or T4 & N1 & M0 \\
IIIc & Any T & N2 & M0 \\
IV & Any T & Any N & M1 \\
\hline
\end{tabular}

Reproduced with permission from O'Connell et al. ${ }^{39}$

M0, no distant metastasis; M1, distant metastasis; N0, no regional lymph node metastasis; N1, metastasis to one to three regional lymph nodes; N2, metastasis to four or more regional lymph nodes; T1, tumor invades submucosa; T2, tumor invades muscularis propria; T3, tumor invades through the muscularis propria into the subserosa or into nonperitonealized pericolic tissues; T4, tumor directly invades other organs or structures and/or perforates the visceral peritoneum.

ment, venous invasion, margin involvement, and tumor perforation-all parameters easily determined by routine pathologic methods. Other factors are more controversial, such as tumor location (left vs. right, i.e., distal or proximal to the splenic flexure) and $\operatorname{sex}^{37}$

\section{Survival of Stage II Colon Cancer Patients According to the New American Joint Committee on Cancer Staging System}

The recently revised 6th edition of the American Joint Committee on Cancer (AJCC) cancer staging system $^{38}$ has increased the stratification of patients with stage II or III colon cancer on the basis of the depth of tumor penetration (T stage) and the number of positive lymph nodes ( $\mathrm{N}$ stage), thus increasing the total number of categories from four to seven (Table 1). A recent study investigated the survival rate of 119,363 patients with colon adenocarcinoma included in the Surveillance, Epidemiology, and End Results US national cancer registry stratified according to the former (5th edition) and current (6th edition) AJCC staging system. ${ }^{39}$ Five-year survival rates in stage II and stage III patients, defined according to the former staging system, were $82.5 \%$ and $59.5 \%$, respectively (Table 2 ). These analyses were performed without considering whether patients received adjuvant chemotherapy. However, stratification according to the new staging system revealed a
TABLE 2. Five-year survival of SEER national registry patients according to the American Joint Committee on Cancer (AJCC) staging system, 6th edition

\begin{tabular}{lccc}
\hline Stage & No. of patients & $\%$ & $P$ value \\
\hline I & 14,500 & 93.2 & - \\
IIa & 28,535 & 84.7 & $<.001$ \\
IIb & 5,826 & 72.2 & $<.001^{a}$ \\
IIIa & 1,989 & 83.4 & $\mathrm{NS}^{b}$ \\
IIIb & 15,946 & 64.1 & $<.001^{c}$ \\
IIIc & 8,600 & 44.3 & $<.001$ \\
IV & 20,802 & 8.1 & $<.001$ \\
\hline
\end{tabular}

Adapted with permission from O'Connell et al. ${ }^{39}$

SEER, Surveillance, Epidemiology, and End Results; NS, not statistically significant.

The $P$ value was determined by the log-rank test and refers to the corresponding stage and the stage in the row above, unless otherwise indicated. All statistical tests were two sided.

${ }^{a}$ IIIa versus IIb.

${ }^{b}$ IIa versus IIIa.

${ }^{c}$ IIb versus IIIb.

significantly lower survival rate in stage IIb patients with T4N0 tumors than in stage IIIa patients with T1 or T2N1 tumors $(72.2 \%$ vs. $83.4 \%$, respectively; $P<$ .001). Admittedly, more patients with stage IIIa colon cancer had received adjuvant chemotherapy than patients with stage IIb in the Surveillance, Epidemiology, and End Results database. The finding of a worse prognosis in patients with stage IIb than stage IIIa disease highlights the need to consider the use of adjuvant chemotherapy in patients with stage II colon cancer, at least in those with poor-prognostic features.

\section{Assessment of the Number of Lymph Nodes}

One of the most important determinants of prognosis in patients with colon cancer is the extent of lymph node involvement. In patients with tumors confined to the bowel wall without lymph node involvement, the 5-year survival rate exceeds $75 \%$, whereas in those with extensive lymph node involvement, it decreases to between $30 \%$ and $60 \% .^{40}$ The likelihood of detecting a positive node increases with the number of nodes examined, so if few lymph nodes are examined, there is an increased risk of a truly stage III patient being mistakenly classified as stage II and possibly being denied the benefit of adjuvant chemotherapy. The possibility of occult stage III patients being included in the population defined as stage II in clinical trials is relevant to any analysis of outcome because it will obscure the relationship between stage and particular treatment regimens. The fact that approximately $25 \%$ to $35 \%$ of patients classified as stage II relapse within 5 years after surgery suggests that the disease may already 
have been disseminated at the time of tumor resection. ${ }^{41}$

The American Joint Committee on Cancer and the tumor-node-metastasis committee of the International Union Against Cancer and the Working Party Report to the World Congresses of Gastroenterology issued the consensus recommendation that at least 12 nodes should be sampled to stage a patient adequately. ${ }^{42}$ Several recent studies have suggested that the number of nodes examined should be between 8 and $20 .^{40,42-45}$ Analysis of the entire patient population included in the Intergroup INT-0089 trial (3411 assessable patients with stage II or III colon cancer) showed that, after controlling for the number of positive nodes invaded, the 5 -year survival rate increased with the number of nodes examined. ${ }^{40}$ This parameter was a highly significant predictor of survival both in patients with lymph node involvement (median number of nodes examined, 13; range, 1-87) and in lymph node-negative patients (median number of nodes examined, 13; range, 1-59).

The prognostic value of the number of lymph nodes analyzed may be explained in various ways. For example, this variable may be a surrogate marker for complete resection of all tumor-bearing tissue for improved intraoperative staging or for improved pathologic staging. In the study described previously, there was no bias in survival owing to the denial of adjuvant chemotherapy to falsely diagnosed nodenegative patients, because all the patients received chemotherapy. In other contexts, this may not be the case, and some institutions now consider the use of adjuvant chemotherapy for stage II patients whenever the pN0 classification is based on the examination of an insufficient number of lymph nodes. This attitude is supported by the recent recommendations on adjuvant chemotherapy for stage II colon cancer published by ASCO, although no absolute number of lymph nodes analyzed was specified as being either adequate or inadequate. ${ }^{46}$

The importance of reliably determining lymph node involvement to achieve accurate tumor staging and the therapeutic implications of this staging have prompted research into detecting the presence of even isolated tumor cells in the lymph nodes analyzed. By using immunohistochemical staining for cytokeratins to detect occult lymph node invasion, it was shown that the presence of isolated tumor cells in mesenteric lymph nodes was an independent predictor of reduced relative survival in patients with stage II colon cancer. ${ }^{41}$ However, many retrospective studies concerning lymphatic mapping have reported contrary findings ${ }^{47}$ The laboriousness and high cost of reliably screening a large number of lymph nodes for occult metastases has led to the concept of sentinel lymph node mapping. The feasibility of sentinel lymph node mapping has been demonstrated for colon cancer, ${ }^{48}$ but its routine clinical use is premature, given the conflicting results published to date. ${ }^{47}$

\section{Molecular Markers}

Molecular (biological) markers provide an additional means of characterizing individual tumors. Several of these markers have been found to influence initial prognosis, and the data suggest that they may influence the response to chemotherapy. Microsatellite instability (MSI), thymidylate synthase expression, aneuploidy, p53 or p21 expression, K-ras mutations, and overexpression or lack of expression of the deleted in colorectal cancer $(D C C)$ gene may be relevant. ${ }^{11}$

One of the most extensively investigated of these markers is MSI, which takes its name from one of the genetic pathways implicated in the development of colorectal cancer. MSI is associated with particular types of mutation that are located in short nucleotide sequences within the chromosome known as microsatellites, which generally result from loss of the DNA mismatch-repair function. MSI occurs in approximately $15 \%$ of colorectal cancers. High-frequency MSI tends to be associated with distinct clinical and pathologic tumor characteristics - e.g., located proximal to the splenic flexure, poor differentiation, mucinous cell type, peritumoral lymphocytic infiltration, and diploidy. The first report that MSI in colon cancers might be associated with a better prognosis was published just over a decade $\mathrm{ago}^{49}$ and has been confirmed by subsequent studies. ${ }^{49,50}$ Halling et al. ${ }^{50}$ examined tumor specimens from 508 patients with resected stage II and III colon cancer and showed by using multivariate analysis that high-frequency MSI was an independent predictor of improved survival and time to recurrence. ${ }^{50}$ Among patients with curatively resected stage II (T3N0M0) colon cancer not receiving adjuvant chemotherapy, Parc et al. ${ }^{51}$ observed a significantly better DFS in those whose tumors presented MSI $(P=.002)$ and they also observed a trend to a probability of a longer OS $(P<.06) .{ }^{51}$ The 5 -year survival rate in this group of high-frequency MSI patients was $>90 \%$, and no recurrence-related death occurred. Age and MSI were found to be independent predictors of a favorable prognosis. This study also demonstrated the feasibility of rapidly screening patients for MSI by using 
an immunohistochemical method suitable for routine use.

The different response to DNA damage of tumors exhibiting high-frequency MSI and those presenting low-frequency MSI or microsatellite stability (MSS) suggests that they may respond differently to chemotherapeutic agents. This hypothesis has been explored in both in vitro and clinical studies. In vitro studies have shown greater resistance to 5-FU in colon cancer cell lines that are deficient in mismatchrepair activity and that have high-frequency MSI compared with cell lines that exhibit MSS. ${ }^{52}$ The results of clinical studies have corroborated these findings. Ribic et al. ${ }^{53}$ examined tumor specimens from 570 patients with resected stage II or III colon cancer who had participated in phase III trials that randomized patients to observation or to adjuvant chemotherapy. ${ }^{53}$ A significantly higher rate of 5-year DFS was observed among the 95 patients $(16.7 \%)$ with tumors exhibiting high-frequency MSI than in patients not presenting this characteristic $(75.3 \%$ vs. $64.1 \%$, respectively; $P=.04$ ). Multivariate analysis adjusting for disease stage and tumor grade also showed a significantly better OS in these patients (HR for death, .61; 95\% CI, .38-.96; $P=.03$ ). Adjuvant chemotherapy significantly increased OS and DFS rates only in patients whose tumors did not exhibit high-frequency MSI. In this group, the HRs for death among treated patients relative to untreated patients were similar in stage II and stage III patients, and there was no evidence of a three-way interaction among treatment effect, MSI status, and stage of disease. Similar results were reported by Carethers et al. ${ }^{54}$ in a series of patients with stage II and III colorectal cancer. ${ }^{54}$ Patients with MSS who received 5-FU had a better survival rate compared with patients who were not treated $(P<.05)$. In contrast, patients with high-frequency MSI who were treated with 5-FU showed no difference in survival compared with patients who were not treated $(P=.52)$.

The conclusions drawn from the results of all these studies suggest that adjuvant chemotherapy may be inappropriate for stage II colon cancer patients with tumors presenting high-frequency MSI, because the prognosis for these patients is already good, and that 5-FU-based adjuvant treatment may be of little or no benefit. In contrast to these data, the NSABP did not find that MSI was a predictive marker for response to 5-FU: patients with MSI (18\%) and patients with MSS cancers were found to benefit equally from the use of adjuvant chemotherapy. ${ }^{55}$ However, prospective validation of MSI as a prognostic factor and/or a predictive factor of chemotherapy efficacy is neces- sary before this parameter is used to guide therapeutic strategy in clinical practice.

Another leading candidate marker is the allelic loss of chromosome $18 \mathrm{q}$, which is associated with tumor progression and is related to the $D C C$ gene. Lanza et al. ${ }^{56}$ observed that patients with stage II cancer and intact 18q alleles had an excellent clinical outcome, with a 5-year DFS of $96 \% .^{56}$ Loss of $18 \mathrm{q}$ alleles in stage III patients with MSS was associated with a 5 -year OS rate after 5-FU-based chemotherapy of $50 \%$ vs. $74 \%$ in those whose tumors retained these alleles. The difference in the relative risk of death was statistically significant $(P=.006) .{ }^{57}$ Zhou et al. ${ }^{58}$ studied 180 patients with stage II colorectal cancer for imbalances of chromosomes $8 \mathrm{p}$ and 18q. The 5 -year DFS was $100 \%$ for patients with no allelic imbalances compared with $58 \%$ for those with allelic imbalances of both chromosomes. ${ }^{58}$ Conversely, Halling et al. ${ }^{50}$ did not find that allelic deletion of $18 \mathrm{q}$ was prognostic in stage II and III colon cancer.

The Cancer and Leukemia Group B 9581 trial for stage II colon cancer (observation vs. monoclonal antibody 17-1A) will include an analysis of both MSI and allelic loss of chromosome 18q, and the correlation of these parameters with outcome will be tested. The planned US Intergroup stage II colon cancer trial will evaluate allelic loss of chromosome $18 \mathrm{q}$ and MSI status in tumor specimens obtained immediately after surgery, and a patient risk profile will be determined by molecular analysis. Patients considered at low risk will be assigned to observation, whereas the high-risk patients will be randomized to 5-FU/FA and oxaliplatin (FOLFOX) or to FOLFOX and bevacizumab.

Numerous other molecular markers have been tested for their ability to predict the prognosis of patients with locally advanced colon cancer after tumor resection. These include expression of the enzymes thymidylate synthase and dihydropyrimidine dehydrogenase, both implicated in resistance of tumors to 5-FU; the cell proliferation marker Ki-67; the apoptotic markers p53 and bcl-2; ploidy; and loss of $18 \mathrm{q}, 17 \mathrm{p}$, and $8 \mathrm{p}$ alleles. Kornmann et al. ${ }^{59}$ found that among patients with stage II or III colon cancer who received adjuvant 5-FU-based chemotherapy, those with high thymidylate synthase levels survived longer than those with low levels of this enzyme. ${ }^{59}$ To derive a more precise estimate of the prognostic significance of thymidylate synthase expression, Popat et al. ${ }^{60}$ reviewed published studies and performed a meta-analysis. The combined HR estimate for OS for low versus high levels was 1.35 (95\% CI, 1.07-1.80) in the adjuvant setting, but there was evidence of 
heterogeneity and possible publication bias. ${ }^{60}$ Additional studies with consistent methodology are needed to define the precise prognostic value of thymidylate synthase. In another study of patients with stage II or III colon cancer (of whom $70 \%$ received 5-FU-based adjuvant chemotherapy), high Ki-67 ( $>27 \%$ ) and diploid status were significantly associated with increased DFS and OS rates in both univariate and multivariate analyses. ${ }^{61}$ Recently, Wang et al. ${ }^{62}$ reported data suggesting that in patients with nonmetastatic colon cancer, allelic imbalance is a better predictor of prognosis than histopathologic stage; they advocated a genomic approach to better define stage II colon cancer prognosis.

\section{CONCLUSIONS}

The widespread use of 5-FU/FA adjuvant chemotherapy regimens for the treatment of patients with stage III locally advanced colon cancers has led to a significant improvement in the prognosis of these patients. The addition of newer agents, such as oxaliplatin, to these regimens has been demonstrated to reduce the probability of relapse still further. ${ }^{19,20}$ The relatively low number of patients with stage II colon cancer included in clinical trials and the low event rate in these patients compared with those with stage III disease have historically led to a lack of power to detect significant treatment benefits in this subgroup. However, newly emerging trials and meta-analyses are reporting a consistent trend toward improved DFS with adjuvant chemotherapy in patients with stage II disease.

The key issue in developing a treatment strategy is the heterogeneous nature of stage II disease, which results in a wide range of postresection prognoses: at one extreme, the prognosis is comparable to that of stage I patients (without unfavorable prognostic factors), for whom the additional benefit adjuvant chemotherapy has not yet been proven, and at the other extreme, it is comparable to that of stage III patients (with unfavorable prognostic factors), for whom the benefit of adjuvant chemotherapy is highly probable. Recognition of this heterogeneity has led to an increasing focus on the factors that influence prognosis and also to an intensive search for reliable prognostic markers and markers predictive of a likely response to adjuvant therapy. Predictive models based on clinical and pathologic variables have already been made available to the oncology community, and the further incorporation into these models of biologic markers, such as MSI and allelic imbalance, will be possible in the near future. Further prospective studies are necessary to validate the prognostic variables proposed, particularly with regard to molecular markers. It should be noted that although all the prognostic factors identified (favorable and unfavorable) have been demonstrated by multivariate analysis, their interaction with the effect of chemotherapy has never been confirmed on a prospective basis.

The recent guidelines published by ASCO on the use of adjuvant therapy, ${ }^{45}$ published before knowledge of QUASAR results, reflect the growing recognition that, although available evidence does not support the routine use of adjuvant chemotherapy in all patients with stage II colon cancer, therapy should be considered for certain poor-prognosis patients (T4 lesions and patients with inadequately sampled nodes, perforation, or poorly differentiated histological characteristics) for whom the likely benefits outweigh the risks and inconvenience of treatment. These ASCO guidelines can help physicians and patients discuss whether to consider adjuvant chemotherapy. This position is supported by recently published data based on the new AJCC staging system showing statistically significantly worse survival in patients with stage IIb cancer (T4N0) than in those with stage IIIa cancer $(\mathrm{T} 1 / 2 \mathrm{~N} 1) .{ }^{39}$ The increasing use of prospective risk stratification in new clinical trials of adjuvant therapy should help to clarify further which stage II patients are likely to gain the most from adjuvant therapy. The results of the QUASAR study, currently reported only in abstract form, are a very important advance toward proving the value of 5-FU-based adjuvant chemotherapy, at least for some stage II patients.

The results observed with oxaliplatin/5-FU suggest that this regimen can further increase the benefit of adjuvant chemotherapy, especially for stage III patients and high-risk stage II patients. At the same time, the availability of more convenient oral fluoropyrimidines - with two studies demonstrating their equivalence to 5-FU/FA bolus regimens in terms of DFS and OS (in stage III patients alone ${ }^{32}$ or plus stage II patients ${ }^{33}$ ) - justifies using these agents as monotherapy in certain patients with stage II disease. The future introduction of targeted therapies, such as cetuximab and bevacizumab, may further enhance the benefit of these treatments. In particular, we must consider the incremental progress as therapies build on past successes. If 5-FUbased therapy provides a $3 \%$ improvement in 5-year DFS compared with no chemotherapy and FOL- 
FOX provides a further $3 \%$ improvement over 5FU, then the absolute benefit of FOLFOX over no treatment may be $5 \%$ to $6 \%$, which would be clinically relevant.

An important question is how large a benefit in OS or DFS is required to achieve not only statistical significance, but also, most importantly, clinical relevance. In the adjuvant setting, and particularly for patients with stage II disease, it is important to consider the potential benefits and risk of treatment. It is crucial to identify reliable prognostic factors and factors predictive of the value of adjuvant therapy, to select the patients for whom this treatment is likely to be advantageous. Several clinical trials are currently under way to evaluate the benefit of adjuvant treatment in patients prospectively defined with poorprognosis (stage II) disease.

In conclusion, according to the data currently available on predictive and prognostic factors, physicians should consider the risks and benefits of adjuvant chemotherapy for stage II patients, making each individual decision for treatment on a case-bycase basis. For patients without risk factors of relapse, the prognosis is comparable to that for stage I, and no data are available to support an additional benefit from adjuvant chemotherapy. For patients with risk factors of relapse (T4 stage, tumor poorly differentiated, tumor perforation, number of lymph nodes examined $<10$ or 12 , or venous invasion), data support an additional benefit from adjuvant chemotherapy. In the near future, therapeutic decisionmaking models are likely to be further refined by the inclusion of molecular markers (e.g., MSI and allelic imbalance). Patients with stage II colon cancer should also be encouraged to participate in randomized clinical trials.

\section{ACKNOWLEDGMENTS}

This article was derived from discussions at a meeting on adjuvant therapy of colon cancer organized by Aimery de Gramont, with financial support from Sanofi-Synthelabo, in September 2003. The authors thank Paula Harry (MediBridge, France) for medical writing support.

\section{REFERENCES}

1. Advanced Colorectal Cancer Meta-analysis Project. Modulation of fluorouracil by leucovorin in patients with advanced colorectal cancer: evidence in terms of response rate. $J$ Clin Oncol 1992; 10:896-903.
2. Levi F, Misset JL, Brienza S, et al. A chronopharmacologic phase II clinical trial with 5-fluorouracil, folinic acid, and oxaliplatin using an ambulatory multichannel programmable pump. High antitumor effectiveness against metastatic colorectal cancer. Cancer 1992; 69:893-900.

3. Giacchetti S, Perpoint B, Zidani R, et al. Phase III multicenter randomized trial of oxaliplatin added to chronomodulated fluorouracil-leucovorin as first-line treatment of metastatic colorectal cancer. J Clin Oncol 2000; 18:136-47.

4. De Gramont A, Figer A, Seymour M, et al. Leucovorin and fluorouracil with or without oxaliplatin as first-line treatment in advanced colorectal cancer. J Clin Oncol 2000; 18:293847.

5. Tournigand C, André T, Louvet C, et al. FOLFIRI followed by FOLFOX6 or the reverse sequence in advanced colorectal cancer: a randomized GERCOR study. J Clin Oncol 2004; 22:229-37.

6. Grothey A, Deschler B, Kroening H, et al. Phase III study of bolus 5-fluorouracil (5-FU)/folinic acid (FA) (Mayo) vs. weekly high-dose $24 \mathrm{~h}$ 5-FU infusion/FA + oxaliplatin (OXA) (FUFOX) in advanced colorectal cancer (ACRC) (abstract 512). Proc Am Soc Clin Oncol 2002; 21:129a.

7. Goldberg RM, Sargent DJ, Morton RF, et al. A randomized controlled trial of fluorouracil plus leucovorin, irinotecan, and oxaliplatin combinations in patients with previously untreated metastatic colorectal cancer. J Clin Oncol 2004; 22:23-30.

8. Hurwitz H, Fehrenbacher L, Novotny W, et al. Bevacizumab plus irinotecan, fluorouracil, and leucovorin for metastatic colorectal cancer. $N$ Engl J Med 2004; 350:2335-42.

9. Cunningham D, Humblet $\mathrm{Y}$, Siena S, et al. Cetuximab monotherapy and cetuximab plus irinotecan in irinotecanrefractory metastatic colorectal cancer. $N$ Engl J Med 2004; $351: 337-45$.

10. Datamonitor. Treatment Algorithms: Colorectal Cancer 5th Edition-New Gold Standards Emerge but Unmet Need Remains. 2003. Available at: http://www.datamonitor.com/ 5316ab639141457fble7238dae8507c9 /industries/research/ ?pid = DMHC1816\&type $=$ Report. Accessed: June 21, 2005.

11. Van Cutsem E, Dicato M, Wils J, et al. Adjuvant treatment of colorectal cancer (current expert opinion derived from the Third International Conference: Perspectives in Colorectal Cancer, Dublin, 2001). Eur J Cancer 2002; 38:1429-36.

12. Compton C, Fenoglio-Preiser CM, Pettigrew N, Fielding LP. American Joint Committee on Cancer Prognostic Factors Consensus Conference: Colorectal Working Group. Cancer 2000; 88:1739-57.

13. Buyse M, Piedbois P. Should Dukes' B patients receive adjuvant therapy? A statistical perspective. Semin Oncol 2001; 28(Suppl 1):20-4.

14. Moertel CG, Fleming TR, Macdonald JS, et al. Levamisole and fluorouracil for adjuvant chemotherapy of resected colon carcinoma. N Engl J Med 1990; 322:352-8.

15. International Multicentre Pooled Analysis of Colon Cancer Trials (IMPACT) investigators. Efficacy of adjuvant fluorouracil and folinic acid in colon cancer. Lancet 1995; 345:93944.

16. O'Connell MJ, Laurie JA, Kahn M, et al. Prospectively randomized trial of postoperative adjuvant chemotherapy in patients with high-risk colon cancer. J Clin Oncol 1998; 16:295-300.

17. Haller DG, Catalano PJ, MacDonald JS, Mayer RJ. Fluorouracil (FU), leucovorin (LV) and levamisole (LEV) adjuvant therapy for colon cancer: five-year report of INT-0089 (abstract 982). Proc Am Soc Clin Oncol 1998; 17:256a.

18. Wolmark N, Rockette H, Mamounas E, et al. Clinical trial to assess the relative efficacy of fluorouracil and leucovorin, fluorouracil and levamisole, and fluorouracil, leucovorin, and levamisole in patients with Dukes' B and C carcinoma of the colon: results from National Surgical Adjuvant Breast and Bowel Project C-04. J Clin Oncol 1999; 17:3553-9. 
19. Andre T, Boni C, Mounedji-Boudiaf L, et al. Oxaliplatin, 5 -fluorouracil and leucovorin as adjuvant treatment of colon cancer: results of the international randomized MOSAIC trial. $N$ Engl J Med 2004; 350:2343-51.

20. Wolmark N, Wieand HS, Kuebler JP, Colangelo L, Smith RE. A phase III trial comparing FULV to FULV + oxaliplatin in stage II or III carcinoma of the colon: results of NSABP protocol C07 (abstract 3500). J Clin Oncol 2005; 23(16 Suppl; Pt 2 of 2):1092s.

21. Mamounas E, Wieand S, Wolmark N, et al. Comparative efficacy of adjuvant chemotherapy in patients with Dukes' B vs. Dukes' C colon cancer: results from four National Surgical Adjuvant Breast and Bowel Project adjuvant studies (C-01, C-02, C-03, and C-04). J Clin Oncol 1999; 17:1349-55.

22. International Multicentre Pooled Analysis of B2 Colon Cancer Trials (IMPACT B2) Investigators. Efficacy of adjuvant fluorouracil and folinic acid in B2 colon cancer. Clin Oncol 1999; 17:1356-63.

23. Liver Infusion Meta-Analysis Group. Portal vein chemotherapy for colorectal cancer: a meta-analysis of 4000 patients in 10 studies. J Natl Cancer Inst 1997; 89:497-505.

24. Gill S, Loprinzi CL, Sargent DJ, et al. Pooled analysis of fluorouracil-based adjuvant therapy for stage II and III colon cancer: who benefits and by how much? J Clin Oncol 2004; 22:1797-806.

25. Adjuvant systemic therapy tools. Available at: http:// www.mayoclinic.com/calcs. Accessed: June 21, 2005.

26. Figueredo A, Germond C, Maroun J, Browman G, WalkerDilks C, Wong S. Adjuvant therapy for stage II colon cancer after complete resection. Provincial Gastrointestinal Disease Site Group. Cancer Prev Control 1997; 1:379-92.

27. Figueredo A, Charette ML, Maroun J, Brouwers MC, Zuraw L. Adjuvant therapy for stage II colon cancer: a systematic review from the Cancer Care Ontario Program in EvidenceBased Care's Gastrointestinal Cancer Disease Site Group. $J$ Clin Oncol 2004; 22:3395-407.

28. Meta-Analysis Group of the Japanese Society for Cancer of the Colon and Rectum and the Meta-Analysis Group in Cancer. Efficacy of oral adjuvant therapy after resection of colorectal cancer: 5-year results from three randomized trials. $J$ Clin Oncol 2004; 22:484-92.

29. Taal BG, Van Tinteren H, Zoetmulder FAN, et al. Adjuvant 5FU plus levamisole in colonic or rectal cancer: improved survival in stage II and III. Br J Cancer 2001; 85:1437-43.

30. Gray RG, Barnwell J, Hills R, et al. QUASAR: a randomized study of adjuvant chemotherapy (CT) vs. observation including 3238 colorectal cancer patients (abstract 3501). Proc Am Soc Clin Oncol 2004; 23:246.

31. Schrag D, Rifas-Shiman S, Saltz L, Bach PB, Begg CB. Adjuvant chemotherapy use for Medicare beneficiaries with stage II colon cancer. J Clin Oncol 2002; 20:3999-4005.

32. Cassidy J, Scheithauer W, McKendrick J, et al. Capecitabine (X) vs. bolus 5-FU/leucovorin (LV) as adjuvant therapy for colon cancer (the X-ACT study): efficacy results of a phase III trial (abstract 3509). J Clin Oncol 2004; 22(14 Suppl):315s.

33. Wolmark N, Wieand S, Lembersky B, Colangelo L, Smith R, Pazdur R. A phase III trial comparing oral UFT to FULV in stage II and III carcinoma of the colon: results of NSABP Protocol C-06 (abstract 3508). J Clin Oncol 2004; 22(14 Suppl):315s

34. Hickish T, Boni C, Navarro M, et al. Stage II patients in the "MOSAIC" trial evaluating oxaliplatin/5-FU/LV as adjuvant treatment of colon cancer: a subpopulation analysis (abstract 284P). Ann Oncol 2004; 15 (Suppl 3):iii76.

35. Sargent DJ, Goldberg RM, Jacobson SD, et al. A pooled analysis of adjuvant chemotherapy for resected colon cancer in elderly patients. $N$ Engl J Med 2001; 345:1091-7.

36. Petersen VC, Baxter KJ, Love SB, Shepherd NA. Identification of objective pathological prognostic determinants and models of prognosis in Dukes' B colon cancer. Gut 2002; 51:65-9.
37. Merkel S, Wein A, Günther K, Papadopoulos T, Hohenberger W, Hermanek P. High-risk groups of patients with stage II colon carcinoma. Cancer 2001; 92:1435-43.

38. AJCC Cancer Staging Manual. 6th ed. New York: SpringerVerlag, 2002.

39. O'Connell JB, Maggard MA, Ko CY. Colon cancer survival rates with the new American Joint Committee on Cancer sixth edition staging. J Natl Cancer Inst 2004; 96:1420-5.

40. Le Voyer TE, Sigurdson ER, Hanlon AL, et al. Colon cancer survival is associated with increasing number of lymph nodes analyzed: a secondary survey of Intergroup Trial INT-0089. $J$ Clin Oncol 2003; 21:2912-9.

41. Bukholm IR, Bondi J, Wiik P, et al. Presence of isolated tumor cells in mesenteric lymph nodes predicts poor prognosis in patients with stage II colon cancer. Eur J Surg Oncol 2003; 29:862-6.

42. Fielding LP, Arsenault PA, Chaphuis PH, et al. Clinicopathological staging for colorectal cancer: an International Documentation System (IDS) and an International Comprehensive Anatomical Terminology (ICAT). J Gastroenterol Hepatol 1991; 6:325-44.

43. Maurel J, Launoy G, Grosclaude P, et al. Lymph node harvest reporting in patients with carcinoma of the large bowel: a French population-based study. Cancer 1998; 82:1482-6

44. Goldstein NS, Weldon S, Coffey M, Layfield LJ. Lymph node recovery from colorectal resection specimens removed for adenocarcinoma: trends over time and a recommendation for a minimum number of lymph nodes to be removed. Am J Clin Pathol 1996; 106:209-16.

45. Wong JH, Severino R, Honnebier MB, Tom P, Namiki TS. Number of nodes examined and staging accuracy in colorectal carcinoma. J Clin Oncol 1999; 17:2896-900.

46. Benson AB, Schrag D, Somerfield MR, et al. American Society of Clinical Oncology recommendations on adjuvant chemotherapy for stage II colon cancer. J Clin Oncol 2004; 22:340819.

47. Zaniboni A, Labianca R, for the Gruppo Italiano per lo Studio e la Cura dei Tumori del Digerente. Adjuvant therapy for stage II colon cancer: an elephant in the living room? Ann Oncol 2004; $15: 1310-8$.

48. Saha S, Dan AG, Beutler T, et al. Sentinel lymph node mapping technique in colon cancer. Semin Oncol 2004; 31:374-81.

49. Thibodeau SN, Bren G, Schaid D. Microsatellite instability in cancer of the proximal colon. Science $1993 ; 260: 816-9$.

50. Halling KC, French AJ, McDonnell SK, et al. Microsatellite instability and $8 \mathrm{p}$ allelic imbalance in stage $\mathrm{B} 2$ and $\mathrm{C}$ colorectal cancers. J Natl Cancer Inst 1999; 91:1295-303.

51. Parc Y, Gueroult S, Mourra N, et al. Prognostic significance of microsatellite instability determined by immunohistochemical staining of MSH2 and MLH1 in sporadic T3N0M0 colon cancer. Gut 2004; 53:371-5.

52. Carethers JM, Chauhan DP, Fink D, et al. Mismatch repair proficiency and in vitro response to 5-fluorouracil. Gastroenterology 1999; 117:123-31

53. Ribic CM, Sargent DJ, Moore MJ, et al. Tumor microsatelliteinstability status as a predictor of benefit from fluorouracilbased adjuvant chemotherapy for colon cancer. $N$ Engl $\mathrm{J}$ Med 2003; 349:247-57.

54. Carethers JM, Smith EJ, Behling CA, et al. Use of 5-fluorouracil and survival in patients with microsatellite-unstable colorectal cancer. Gastroenterology 2004; 126:394-401.

55. Allegra CJ, Kim G, Kirsch IR. Microsatellite instability in colon cancer. $N$ Engl J Med 2003; 349:1774-6.

56. Lanza G, Matteuzzi M, Gafá R, et al. Chromosome 18q allelic loss and prognosis in stage II and III colon cancer. Int J Cancer 1998; 79:390-5.

57. Watanabe T, Wu TT, Catalano PJ, et al. Molecular predictors of survival after adjuvant chemotherapy for colon cancer. $N$ Engl J Med 2001; 344:1196-206. 
58. Zhou W, Goodman SN, Galizia G, et al. Counting alleles to predict recurrence of early-stage colorectal cancers. Lancet 2002; 359:219-25.

59. Kornmann M, Schwabe W, Sander S, et al. Thymidylate synthase and dihydropyrimidine dehydrogenase mRNA expression levels: predictors for survival in colorectal cancer patients receiving adjuvant 5-fluorouracil. Clin Cancer Res 2003; 9:411624.

60. Popat S, Matakidou A, Houlston RS. Thymidylate synthase expression and prognosis in colorectal cancer: a systematic review and meta-analysis. J Clin Oncol 2004; 22: 529-36.

61. Garrity MM, Burgart LJ, Mahoney MR, et al. Prognostic value of proliferation, apoptosis, defective DNA mismatch repair, and p53 overexpression in patients with resected Dukes' B2 or C colon cancer: a North Central Cancer Treatment Group study. J Clin Oncol 2004; 22:1572-82.

62. Wang Y, Jatkoe T, Zhang Y, et al. Gene expression profiles and molecular markers to predict recurrence of Dukes' B colon cancer. J Clin Oncol 2004; 22:1564-71. 